\title{
Plant Invasions of New Zealand with Reference to Lord Howe, Norfolk, and the Kermadec Islands.
}

\author{
BY \\ J. C. WILLIS, M.A., Sc.D., F.R.S., \\ European Correspondent of the Botanic Garden, Rio de Janeiro.
}

With three Diagrams and eleven Tables in the Text.

I $\mathrm{N}$ a series of eight papers in this journal, IyI6-I9, I have dealt with the 1 floras of New Zealand proper and the islands which outlie from it to the north (Kermadecs), east (Chathams), and south (Stewart and Aucklands), islands which must have received a large part of their flora either directly from New Zealand or from invasions which passed near to them on the way thither. In these papers I have made a great many predictions based upon my hypothesis of age and area, and have always found them borne out by the facts. It is almost needless to say that in some cases these facts were already known to New Zealand and other botanists, though very many are new. My chief object in making all these predictions was to marshal the facts and to show (and I venture to think that $I$ have shown) that age and area can be relied upon as a guide in the taxonomic distribution problems of a well-defined area like New Zealand and its immediately outlying islands. I shall now go farther afield, and endeavour to trace farther towards their source some of the invasions of plants which appear to have reached New Zealand from the north or north-west.

One of the principal objects kept in view in this work is to show that the floras of the islands which lie between New Zealand and Australia or Polynesia fit in with my hypothesis of age and area, and are explicable on that hypothesis; further, that they are also capable of being dealt with by aid of numerical methods, like the floras of New Zealand itself, and, therefore, that the invasions of plants must have been by land-casual transaqueous carriage would not produce such results.

In the first papers of this series, dealing with Ceylon (7), by means of statistics of actual distribution of the various species, which were taken from Trimen's Flora, and which showed that the least widely distributed species in the island were those confined to Ceylon, the next those confined to Ceylon and South India, and the most widely distributed in the island those

[Annals of Botany, Vo1. XXXIV. No. CXXXVI, October, 1920.] 
which had a greater distribution abroad than merely to South India, I deduced the conclusion that in a given country the area of distribution of a species (working always with groups of at least ten allied species) depended upon its age in that country. The correctness of this deduction was then confirmed by observations upon the floras of New Zealand and its surrounding islands, which gave exactly parallel results, and also by observations on the floras of Hawaii, Jamaica, \&c.

In my first paper on the New Zealand flora (9), I assumed the correctness of the hypothesis (which for convenience I have termed that of 'age and area '), and by means of the diagram here reproduced I predicted the result

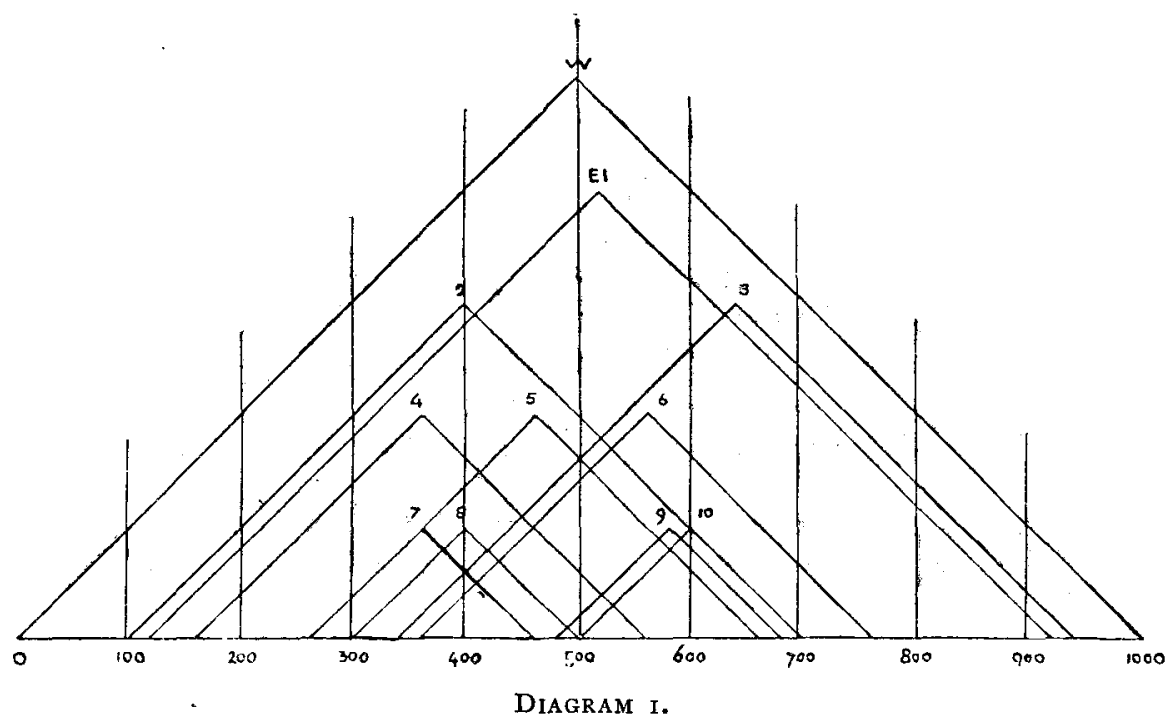

of the entrance of a single species (w) at the centre of New Zealand, which spread at a uniform rate (represented by the triangle), and which subsequently gave rise to local endemic species ( $E$ I to $E$ IO) in a casual way. The length of New Zealand, supposed I,000 miles, being divided into ten zones of equal width, and the number of endemics found in each zone being counted (each endemic of course being supposed to spread uniformly like the parent species, as indicated by the similar triangles), the result shows a curve rising and falling to and from a maximum (sometimes two) which is somewhere near the point of entry of the original species. In the present case, for example, the numbers of endemics in each consecutive zone are-

$$
\begin{array}{llllllllll}
\circ & 3 . & 5 & 8 & 9^{1} & 8 & 7 & 3 & 2 & 2
\end{array}
$$

It will be well to make clear at this point that if the entry of the original species be at one end, instead of in the middle of New Zealand, the curve will in general show its maximum at or near that end. The maximum,

1 The thicker type indicates the maximum in this and the following table. 
in other words, is in general at or near the point of entry of the original species. Further, it is not necessary that the entry should be at a point. If it were, for example, by the whole zone from 300 to 700 miles, the -resulting curve would be of the same type.

Examination of the actual figures for the distribution of the New Zealand flora soon showed that every genus did in fact give a curve of this type, so that a fact of great significance was thus discovered, and one which may be considered without reference to the hypothesis of age and area, by whose assistance it was originally found. The distribution of the numbers followed two general types, as seen in the examples quoted below:

TABLE I.

\begin{tabular}{|c|c|c|c|c|c|c|c|c|c|c|c|}
\hline & $0-100 \mathrm{~m}$. & $I-200$ & $2-300$ & $3-400$ & $4-500$ & $5^{-600}$ & $6-700$ & $7-800$ & $8-900$ & $9-1000$ & $10-1080$ \\
\hline Ranunculus & . - & 2 & 3 & 5 & 7 & I I & 12 & 18 & 18 & to & 2 \\
\hline Drimys . & & 2 & 2 & 2 & 2 & 3 & 3 & 2 & I & I & I \\
\hline Pittosporum & .11 & 21 & 11 & 11 & 8 & 7 & 6 & 6 & 5 & 5 & I \\
\hline Colobanthus &.- & - & - & - & - & 2 & 3 & 3 & 4 & 2 & - \\
\hline Coprosma. & . 12 & 12 & 15 & 16 & I 7 & 18 & 18 & 16 & I5 & 12 & 3 \\
\hline Metrosideros & s. 8 & 8 & 8 & 8 & .5 & 6 & 6 & 2 & I & $r$ & I \\
\hline Ligusticum & . - & I & $\mathbf{I}$ & I & 2 & 7 & 8 & 9 & 7 & 6 & 3 \\
\hline Veronica. & . 6 & 6 & IO & $I_{4}$ & I5 & 39 & $4^{I}$ & 43 & $3^{8}$ & 26 & 2 \\
\hline Utricularia & 3 & $\mathbf{3}$ & 3 & I & $\mathrm{I}$ & I & I & I & - & - & - \\
\hline Pimelea. & 4 & 4 & 5 & 5 & 7 & 8 & 8 & 6 & 5 & 4 & I \\
\hline
\end{tabular}

In all cases the figures show a maximum with a regular falling off, but in some, e.g. Pittosporum, the maximum is to the north, with no falling off to northwards, in others, e.g. Ranunculus, it is further south, with a falling off in both directions, and, as the examples chosen illustrate, the maximum may be at any zone from 5-600 to 8-900 miles. This phenomenon of a simple curve to a maximum (or two) is shown by every genus in the flora.

These curves represent simply the naked unvarnished facts of taxonomic distribution, and they may be considered without reference to my hypothesis of age and area. It is quite clear from them that, for example, the previous distributional history of Pittosporum was different from that of Ranunculus or Veronica. And it is equally clear that in broad outlinewhich is all that we are as yet concerned with in taxonomic distributionthat distribution was not determined by biological agents, but by a more purely mechanical cause.

Not onlyso, but it is also extremely difficult to believe that such regularity as this, and regularity of two types, would be shown as the result of casual arrivals across a wide expanse of water. It is hard to believe that it can be explained-again in broad outline, and to the extent of say 90 per cent. of the flora-by anything but a previous land connexion of New Zealand with Indo-Malaya, and with other sources of flora. My critics, who frequently insist upon applying age and area to individual cases, force upon me the opinion that neither biological agencies nor casual distribution across the sea have had any hand in the present distribution of the New Zealand flora. 
This, however, is not my own opinion; what I maintain is that in about $9 \circ$ per cent. or perhaps more of the cases, the distribution has been by land, and has been chiefly mechanical, so far as its broad outline is concerned. Age and area must not be applied to individual cases.

A nother point whose misunderstanding is frequently a stumbling-block in the way of acceptance of age and area, is a confusion of the two types of distribution known as taxonomic and ecological. The former, with which alone age and area is concerned, takes no account of the density or rarity of a given species upon the ground, but simply of the total area over which it is found, while the latter is much more concerned with the density or rarity of a given species under a given set of ecological conditions. From the taxonomic or age and area point of view there is no difference between the species represented by the two groupings of letters below :
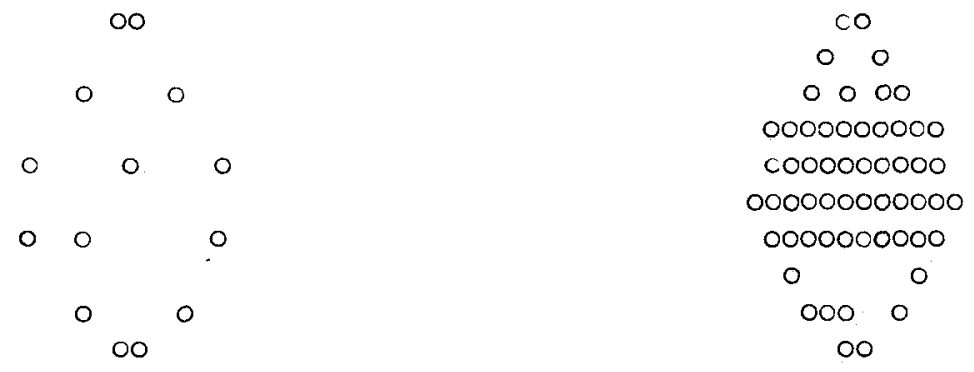

and in this connexion it is a pleasure to acknowledge the justice and value of Mrs. Arber's criticism (1), while drawing attention to the fact that she incidentally refers to, that people have in general regarded species with small areas as species that are dying out (cf. 10, p. 349). If this can no longer be accepted, then my general contention that endemics of small area are not necessarily relics of past floras, but are usually young species that have not had time to spread, receives very strong support.

Examining these zonation figures in detail, one at once finds that a great many genera show their maximum at the far north of New Zealand, like Pittosporum, Metrosideros, and Utricularia in Table I* They do not show any falling off to the northwards, even though the figures may be the same for the first two, three, or even four zones. Now a consideration of Diagram I, given on p. 472 , will show that the maximum of endemics must in general occur somewhere near to the point or zone of original entry of the first species to arrive from abroad. Consequently when one deals, as is now the case, with a large number of different genera, it is clear that the variations to one side or the other will cancel one another, and, therefore, that the region of entry into New Zealand (as now limited by the ocean) was within the first 300 or at most 400 miles of the northern part of the North 
Island. These species with northern entry are fairly easily segregated from the rest of the flora.

As already indicated in (10), p. 356, Table I, we may look upon about thirty-three families of the ninety-one of the New Zealand flora as showing this northern maximum. But it would be a great mistake to assume that all members of these families entered from the north. So many of them show a northern maximum that their figures swamp those of any that may have entered in any other way, but a more satisfactory result is obtained by going through the figures genus by genus. Even then one finds genera in which there are some wides with evident northern entry, and others with equally evident entry by some other route, but these are few in comparison with the total, and for the present it is better not to attempt to go into too minute detail.

Examining the whole of the genera of the New Zealand flora as given by Cheeseman (all the larger of them are given in Tables $\mathrm{V}$ and VI of (9)), I have arrived at the general tentative result given in Table II below. While the northern types seem to divide into a northern (proper) and a Kermadec invasion $(11$, p. 279), the rest seem to nie to split into a western and a southern invasion, a considerable number (western) having their centre of greatest density between $4-500$ and 6-700 miles from North Cape; for instance, Drimys, Coprosma, and

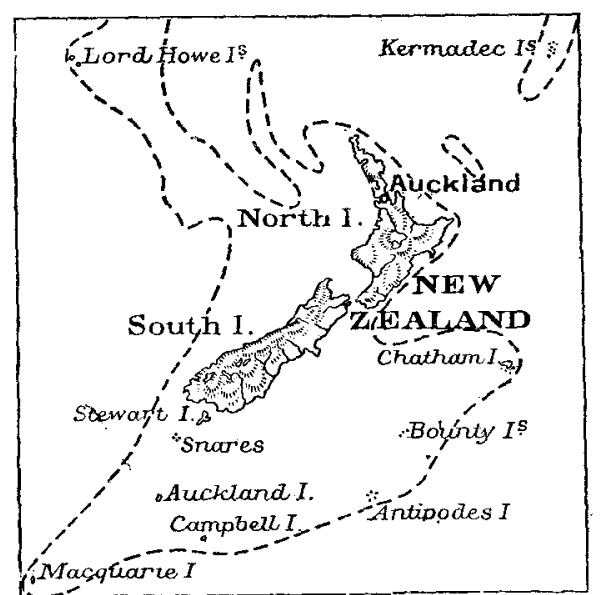

Diagram 2. New Zealand and ontlying islands. The dotted line is the $I, 000$ fathom limit. Pimelea in Table I. In such cases the very marked tapering off of the numbers both to north and to south makes it fairly probable that they must have commenced at the middle, but these genera run imperceptibly into those of the more southern group which we have already considered in a previous paper, so as to render it very difficult in many cases to separate them. A glance at the map, showing the broadly triangular way in which the water of less than 1,000 fathoms meets the west coast of New Zealand, will help to make it more clear why this should be so. But I am inclined to think, none the less, that my figures indicate that there were probably two different invasions, a western and a southern. At the time when the greater part of the sea floor to I,000 fathoms was dry land, it is clear that a species arriving at the point where stands the $\mathrm{N}$ of 'North I.' would be able to spread out fanwise, and might ultimately reach (the existing) New Zealand along a wide front. 
There is so distinct a group with maxima in the far south, comprising a great part of eighteen families (10, p. 359), that I am inclined to think that they formed a separate southern invasion, but as $I$ have pointed out $\left(10,3^{6} \mathrm{I}\right)$, the origin of this invasion is a complex question that must be left for the present unanswered, though I am inclined to put it down to Antarctica. It is conceivable, though improbable, that its route was along the southern side of the bridge by which the western invasion probably arrived.

There follows a complete list of the New Zealand genera, giving in each case the invasion to which $I$ incline to attribute it, whether northern $(N)$, northern by the Kermadecs $(\mathrm{K})$, western $(\mathrm{W})$, southern $(\mathrm{S})$, and giving also the northern islands, Kermadecs $(\mathrm{K})$, Norfolk $(\mathrm{N})$, or Lord Howe $(\mathrm{H})$, on which they occur.

\section{TABI.E II.}

The first entry after the name of a genus, e. g. SW, refers to the invasion or invasions (Northern, Kermadec, Western, or Southern) to which the general distribution of its endemics (and wides when they do not range the whole length of New Zealand) points.

A ? after a letter refers only to that letter, e.g. SW ? means pretty certainly $S$ and perhaps $W$.

The second entry $(\mathrm{K}, \mathrm{N}, \mathrm{H})$ refers to the islands (Kermadecs, Norfolk, Howe) in which the genus is found.

Genera in brackets (in small letters) occur only in the Kermadecs in the New Zealand area.

Genera in capitals are endemic; if in brackets endemic in the Chathams or Aucklands.

\section{DICOTYLEDONS}

1. Ranunculaceae

1. Clematis

2. Myosurus

3. Ranunculus

4. Caltha

2. Magnoliaceae

\section{Drimys 3. Cruciferae}

6. Nasturtium

7. Cardamine

9. PACHYCLADON

10. Capsella

I . Lepidium

12. NOTOTHLASPI

I3. Viola 4. Violaceae

14. Melicytus

I5. Hymenanthera 5. Pittosporaceae

16. Pittosporum
6. Caryophyllaceae

17. Gypsophila

18. Stellaria

19. Colobanthus

20. Spergularia 7. Portulacaceae

21. Claytonia

22. Montia

23. HECTORELLA 8. Elatinaceae

24. Elatine
9. Hypericaceae

25. Hypericum Io. Malvaceae

26. Plagianthus

$\begin{array}{ll}\text { Invasion. } & \text { Islands. } \\ \text { W } & \mathrm{NH} \\ \text { W } & - \\ \text { SW } & \mathrm{N} \\ \text { WS } & - \\ \text { WN } & \mathrm{H} \\ \mathrm{W} ? & \mathrm{~N} \\ \mathrm{~S} & \mathrm{~K} \\ \mathrm{~W} & - \\ ? & \\ \text { SW } & \mathrm{H} \\ \mathrm{WS} & \mathrm{N} \\ \mathrm{WN} & \mathrm{KN} \\ \mathrm{W} & \mathrm{NH} \\ \mathrm{N} & \mathrm{KNH} \\ \mathrm{W} ? & - \\ \text { SW } & - \\ \mathrm{S} & - \\ ? & - \\ \mathrm{S} & - \\ \mathrm{S} & - \\ & - \\ ? & - \\ \text { W } & - \\ \mathrm{W} ? & -\end{array}$

DICOTYLEDONS Invasion. Islands.

27. HOHERIA

28. Gaya

29. Hibiscus

I I. Tiliaceae

30. ENTELEA

31. Aristotelia

32. Elaeocarpus

I2. Linaceae

33. Linutm

I3. Geraniaceae

34. Geranium

35. Pelargonium

36. Oxalis

I4. Rutaceae

37. Phebalium

38. Melicope I5. Meliaceae

39. Dysoxylum I6. Olacineae

40. Pennantia 17. Stackhousiaceac

41. Stackhousia

18. Rhamnaceae

42. Pomaderris

43. Discaria 19. Sapindaceae

44. Dodonaea

45. Alectryon 20. Anacardiaceae

46. Corynocarpus 2I. Coriariaceae

47. Coriaria 22. Leguminosae

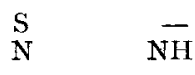

W?

W?

W?

W ?

W?

$\mathrm{W} ? \quad \mathrm{KH}$

$\mathrm{N}$

$\mathrm{N} \quad \mathrm{KH}$

$\mathrm{N}$

$\mathrm{NH}$

NW

$\mathrm{N}$

W

$\mathrm{N}$

$\mathrm{W}$ ?

$\mathbf{N}$

$\mathrm{NH}$

$\mathrm{N} \quad$

KW K

WN K

48. CORALLOSPAR TIUM

49. Carmichaelia

WS

5o. NOTOSPARTIUM 


\begin{tabular}{|c|c|c|c|c|c|}
\hline DICOTYLEDONS & Invasion. & Islands. & DICOTYLEDONS & nvasion. & Islands \\
\hline Clianthus & $\mathbf{N}$ & $N$ & 103. Galium & W ? & 一 \\
\hline $\begin{array}{l}\text { 2. Sroainsona } \\
\text { 3. (Canazalia) }\end{array}$ & W & $(\overline{\mathbf{K N H}})$ & $\begin{array}{l}\text { I04. Asperula } \\
3^{8 .} \text {. Compositae }\end{array}$ & W ? & - \\
\hline Sophora & W? & $\mathrm{H}$ & 105. (Ageratum) & & $(\mathbf{K})$ \\
\hline 23. Rosaceae & & & ro6. Lagenophora & W ? & $\mathrm{K}$ \\
\hline Rubus & $W ?$ & & 107. Brachycome & SW & $\mathbf{H}$ \\
\hline Geum & $\mathrm{W} ? \mathrm{~S} ?$ & - & 108. Olearia & W & $\mathbf{H}$ \\
\hline Potentilla & W? & 一 & \multicolumn{3}{|l|}{ rog. (PLEUROPHYLLUM) } \\
\hline Acaena & $?$ & $\mathrm{~K}$ & I ro. Celmisia. & $\mathrm{S} ?$ & 一 \\
\hline 24. Saxifragaceae & & & II. Vittadinia & W? & - \\
\hline 9. Donatia & $S$ & 一 & I 12. $H A A S T I A$ & & \\
\hline Quintinia & WN? & - & I13. Gnaphalium & WS? & $\mathrm{KNH}$ \\
\hline$I X E R B A$ & & & I I 4. Raoulia & $S ?$ & - \\
\hline CARPODETUS & & & II 5. Helichrysum & W & - \\
\hline 3. Ackama & $N$ & - & II6. Cassinia & W & $\mathrm{H}$ \\
\hline $\begin{array}{l}\text { 4. Weinmannia } \\
\text { 25. Crassulaceae }\end{array}$ & W ? & - & $\begin{array}{l}\text { I 17. Craspedia } \\
\text { I } 8 \text {. Siegesbeckia }\end{array}$ & $\begin{array}{l}\mathrm{W} ? \\
\mathrm{~K}\end{array}$ & $\overline{\mathbf{K}}$ \\
\hline $\begin{array}{l}\text { 5. Tillaea } \\
\text { 26. Droseraceae }\end{array}$ & WS ? & - & $\begin{array}{l}\text { I19. Bidens } \\
\text { I 20. Cotula }\end{array}$ & $\begin{array}{l}\mathrm{K} \\
\mathrm{SW}\end{array}$ & $\begin{array}{l}\text { KNH } \\
\text { KNH }\end{array}$ \\
\hline $\begin{array}{l}\text { 66. Drosera } \\
\text { 27. Haloragidaceae }\end{array}$ & NWS? & - & $\begin{array}{l}\text { 121. Centipeda } \\
\text { I22. Abrotanella }\end{array}$ & W ? & - \\
\hline 7. Haloragis & $?$ & $\mathrm{~K}$ & 123. Erechtites & WS & $\mathrm{NH}$ \\
\hline 8. Myriophyllum & $?$ & - & $124 . B R A C H Y G L O T T T S$ & & \\
\hline 9. Gunnera & S ? & - & I25. Senecio & W & $\mathrm{KNH}$ \\
\hline Callitriche & $?$ & $\mathrm{~K}$ & I 26. Microseris & W? & - \\
\hline 28. Myrtaceae & & & I27. Picris & $\mathrm{N}$ ? & $\mathbf{N}$ \\
\hline 7. Leptospermum & NW & $\mathrm{H}$ & 128. Crepis & $\mathrm{S} ?$ & - \\
\hline 72. Metrosideros & $\mathrm{N}$ & KH & I29. Taraxacum & W? & - \\
\hline 3. Myrtus & $\mathbf{N}$ & - & 130. Sonchus & W? & $\mathrm{KNH}$ \\
\hline 74. Eugenia & $\mathrm{N}$ & 一 & 39. Stylidiaceae & & \\
\hline $\begin{array}{l}\text { 29. Onagraceae } \\
\text { Exilobium }\end{array}$ & SW? & 一 & $\begin{array}{l}\text { '3!. Phyllachne } \\
\text { OSEOST YLIDITI }\end{array}$ & $S$ & - \\
\hline $\begin{array}{l}\text { 76. Fuchsia } \\
\text { 3o. Passifloraceae }\end{array}$ & $?$ & 一 & $\begin{array}{l}\text { I33. Forstera } \\
\text { 40. Goodeniacene }\end{array}$ & $S ?$ & 一 \\
\hline $\begin{array}{l}\text { 77. Passiflora } \\
\text { 3. Cucurbitaceae }\end{array}$ & $\mathbf{N}$ & $\mathrm{NH}$ & $\begin{array}{l}\text { I34. Selliera } \\
\text { I } 35 . \text { Scaevola) }\end{array}$ & W ? & $\overrightarrow{(\mathbf{K})}$ \\
\hline $\begin{array}{l}\text { 78. Sicyos } \\
3^{2} \text {. Ficoideae }\end{array}$ & $\mathrm{KN}$ & $\mathrm{KNH}$ & $\begin{array}{l}\text { 4r. Canipanulaceae } \\
\text { 136. COLENSOA }\end{array}$ & & \\
\hline 79. Mesembryanthemum & NW & $\mathrm{KNH}$ & I37. Pratia & WS? & - \\
\hline 8o. Tetragonia & NW & KNH & I38. Lobelia & WS? & $\mathrm{KNH}$ \\
\hline $\begin{array}{l}\text { 33. Umbeiliferae } \\
\text { Iydrocotyle }\end{array}$ & ד & $\mathrm{KH}$ & I39. Isotoma & SW? & $\overline{\mathrm{KNH}}$ \\
\hline $\begin{array}{l}\text { 81. Hyarocotyle } \\
\text { 82. Azorella }\end{array}$ & $\begin{array}{l}\text { W } \\
\mathrm{S} ?\end{array}$ & KH & $\begin{array}{l}\text { I40. Wahlenbergata } \\
\text { 42. Ericaceae }\end{array}$ & & \\
\hline 83. Eryngium & W & - & I4I. Gaultheria & W & - \\
\hline 84. Actinotus & $S$ & - & I42. Pernetiya & $\mathrm{S}$ & 一 \\
\hline 85. Apium & $\mathrm{N}$ ? & KNH & 43. Epacridaceae & & \\
\hline 86. Oreomyrrhis & $\mathrm{W}$ & - & 143. Pentachondra & $\mathrm{W} ?$ & \\
\hline 87. Crantzia & $W ?$ & - & 144. Cyathodes & SW & - \\
\hline 88. Aciphylla & $\mathrm{SW} ?$ & - & I 45. Leucopogon & NW? & $\mathrm{H}$ \\
\hline 89. Ligusticum & $\mathrm{S}$ & - & 146. Epacris & $\mathbf{W} ?$ & - \\
\hline 9o. Angelica & $\mathrm{S} ?$ & 一 & 147. Archeria & W? & - \\
\hline 9. Daucus & W? & - & I 48. Dracophyllum & W & $\mathrm{H}$ \\
\hline 92. STILBOCARPA & & & 149. Samolus & $?$ & $\mathrm{KN}$ \\
\hline 93. Aralia & S & & 45. Myrsinaceae & & \\
\hline 94. Panax & $\begin{array}{l}W \\
N\end{array}$ & $\begin{array}{l}\mathrm{KH} \\
\mathrm{N}\end{array}$ & ${ }^{1} 50$. Myrsine (incl Rapanea) & $W ?$ & KNH \\
\hline $\begin{array}{l}\text { 96. Schefflera } \\
\text { 97. PSELUDOPANAX }\end{array}$ & $?$ & - & $\begin{array}{l}\text { 46. Sapotaceae } \\
\text { I5 I. Sideroxylon }\end{array}$ & $\mathrm{N}$ & NH \\
\hline 98. COROKIA & & & I52. Olea & $\mathrm{N}$ & NH \\
\hline $\begin{array}{l}\text { 99. Griselinia } \\
3^{6} \text {. Caprifoliaceae }\end{array}$ & $\mathrm{W} ?$ & & $\begin{array}{l}\text { 48. Apocynaceae } \\
\text { 153. Parsonsia }\end{array}$ & W? & \\
\hline oo. Alsenosmia & $\mathbf{N}$ & 一 & 49. Loganiaceae & & \\
\hline 37. Rubiaceae & & & I54. Mitrasacme & $?$ & - \\
\hline o1. Coprosma & W & $\mathrm{KNH}$ & I55. Logania & $?$ & - \\
\hline o2. Nertera & NWS? & $\cdots$ & 156. Geniostonia & $\mathrm{N}$ & $\mathrm{H}$ \\
\hline
\end{tabular}


DICOTVLEDONS 50. Gentianaceae

I57. Sebaed
I58. Gentiana
159. Liparophyllum
5. Boraginaceae
160. Myosotis
I61. (MVOSOTIDIUM)
I62. TETRACHONDRA
52 . Convolvulaceae 163. Iponoea

164. Calystegia

165. Convoluulus

166. Dichondra

167. Cuscuta 53. Solanaceae

168. Solànum 54. Scrophulariaceae

169. Calceolaria

I7o. Mimulus

r71. Mazus

I72. Gratiola

I73. Glossostigma

174. Limosella

175. Veronica

176. Ourisia

I77. Euphrasia

г78. $A N A G O S P E R M A$

179. SIPIIONIDIUM

55. Lentibulariaceae

I80. Utricularia 56. Gesneriaceae

18 ז. RIIABDOTIIAMNUS 57. Myoporaceae

182. Myoporum 58. Verbenaceac

183. Vitex

184. TEUCRIDIUM

I8. Avicennia

186. Mentha 59. Labiatae

I87 Scutellaria 6o. Plantaginaceae

I88. Plantago 6r. Nyctaginaceae

I89. Pisonia 62. Illecebraceae

19o. Scleranthus 63. Amarantacene

I9I. Alternanthera 64. Chenopodiaceae

192. Rhagodia

I93. Chenopodium

194. Atriplex.

I95. Salicornia

I96. Suacda

197. Salsola 65. Polygonaceae

198. Polygonum

199. R'umex

2oo. Muehlenbeckia 66. Piperaceae

201. Piper

202. Peperomia 67. Chloranthaceae

203. Ascarina 68. Monimiaceae

204. Hedycarya
Invasion. Islands.

$\begin{array}{ll}\text { W? } & - \\ \text { WS } & - \\ \text { S? } & - \\ \text { WS } & - \\ & \\ ? & \text { KNH } \\ \text { WN? } & \text { KNH } \\ \text { W? } & - \\ \text { W? } & - \\ ? & -\end{array}$

$\mathrm{W}$ ?

W?

W ?

W ?

W?

W?

W?

$\mathrm{S}$

$\mathrm{S}$

WS ?

KNH

$=$
$\overline{-}$
$\frac{\mathrm{KN}}{}$

NWS?

NW

N

$\mathrm{N}$

W?

W

SW

$\mathrm{N}$

W?

N

K

WS?

W?

W

W

W

W ?

$?$

W?

K

$\mathrm{K}$

$?$

$\mathrm{N}$ ?

N

$\mathrm{NH}$

H

II

KNH

KNH

KNH

K
DICOTYLEDONS 205. Laurelia

60. Lauraceae

206. Beilschmiedia

207. Litsaea

208. Cassytha

7o. Proteaceae

209. Persoonia

210. Knightia

7.. Thymelaeaceae

211. Pimslea
212. Drapetes
72 . Loranthaceae
213. Loranthus

214. TUPEIA

2I5. Viscum

73. Santalaceae

Invasion.

Tslands.

216. Fusanus

$\mathrm{N}$ ?

$\mathrm{N}$

$\mathrm{N}$

$\mathrm{N}$

$\mathrm{N}-$

W

$\mathrm{S}$ ?

H

WS

WS

NH

217. Exocarpus

N

$\overline{\mathrm{NH}}$

218. $D A C T Y L A T T H C$

75. Euphorbiaceae

2I9. Euphorbia

20. Poranthera

22 I. (Alewrites)

22. (Homalanthus)

76. Urticaceae

23. Paratrophis

224. Uritica

225. Eilatostema

226. (Boehneria)

227. Parietaria

228. Australina

77. Cupuliferae

KNH 229. Fagus

$\stackrel{?}{W}$ ?

NH

(K)

(K)

$N ?$

$\mathrm{SW}$ ?

$\mathrm{N}$

H

N

(KNH)

W?

W?

MONOCOTVLEDONS

78. Orchidaceae

230. Dendrobium

23 I. Bulbophyllum

232. Earina

233. Sarcochines

234 . Spiranthes

H 235. Thelymitra

236. Orthoceras

237. Microtis

238. Prasophyllum

239. Caleana

240. Pterostylis

24I. Acianthus

242. Cyrtostylis

243. Calochilus

244. Lyperanthus

245. Caladenia

246. Chiloglottis

247. Adenochilus

248. TOWNSONIA

249. Corysanthes

250. Gastrodia

79. Iridaceae

251. Libertia

8o. Amaryllidaceae

252. Hypoxis

81. Liliaceae

253. Rhipogonum

$?$

N?

$N$ ?

$\mathrm{N}$

N

$\mathrm{N}$

$\mathrm{N}$ ?

?

W

K

N

$\mathrm{N}$ ?

$\mathrm{S}$ ?

WS?

$\mathrm{W}$ ?

W?

$N$ ?

NW

W?

W ?

W ?

W?

$\mathrm{N}$ ?

255. Cordyline

256. Astelia
$\mathrm{N}$

NII

NH

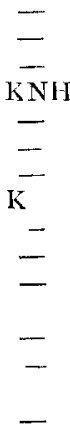

NH

$\mathrm{KN}$ 
MONOCOTYLEDONS Invasion.

257. Dianella
258. Phormium
259. Bulbinella
260. Arthropodium
261. Herpolirion
262. Iphigenia
82. Juncaceae
263. Rosthovia
264. Funcus
265. Luzula
83. Palmae
266. Rhopalostylis
84. Pandanaceae
267. Freycinetia
85. Typhaceae
268. Typha
269. Sparganium
86. Lemnaceae
270. Lemna
87. Naiadaceae
271. Triglochin
272. Potamogeton
273. Ruppia
274. Zannichellia
275. Lepilaena
276. Zostera
88. Centrolepidaceae
277. Trithuria
278. Centrolepis
279. Gaimardia
89. Restionaceae
280. Lepyrodia
281. Leptocarpus
282. Hypolaena
90. Cyperaceae
283. Kyllinga
284. Cyperus
285. Mrariscus
286. Eleocharis
287. Fimbristylis
288. Scirpus
289. Carpha

$\begin{array}{ll}\mathrm{N} ? & \mathrm{NH} \\ ? & \mathrm{~N} \\ ? & - \\ \stackrel{\mathrm{W}}{\mathrm{S}} & - \\ \mathrm{S} ? & -\end{array}$

MONOCOTYLEDONS Invasion.

Islands.

9o. Schoenus

291. Cladium

292. Lepidosperma

293. Gahnia

294. Oreobohus

295. Uncinia

296. Carex

SW $\quad$ H $\quad$ 297. Imperata

$\mathrm{S} \quad \mathrm{H}$ 298. Zoysia

299. Paspalum

$\mathrm{N} \quad \mathrm{KN}$ 300. Isachne

301. (Panicum)

302. Oplismenus

303. (Cenchrus)

304. Spinifex

305. Ehrharta

306. Microlaena

307. Hierochloe

W? $\quad-\quad$ 308. Stipa
$\mathrm{W} ? \quad$ 309. Echinopogon

W? - 310. Alopecurus

$-\quad$ 3II. Sporololus
312. SIMPLICIA

3II. Sporololus
312. SIMPLICIA

W

$? \quad$ ? $\quad$ - $\quad \begin{aligned} & 3^{13} \cdot \text { Agrostis } \\ & 3^{14} \text {. Deyeuxia (Calama } \text { orostis) }\end{aligned}$

$\stackrel{\mathrm{WN}}{\mathrm{N}} \mathrm{H}$

$\mathrm{N} \quad \ldots$

WN $\quad \mathrm{H}$

$\stackrel{?}{\mathrm{SW}} \quad \overrightarrow{\mathrm{H}}$

SW $\quad$ KNH

$\mathrm{N} \quad \mathrm{K}$

$\mathrm{N} \quad \overline{\mathrm{NH}}$

$\mathrm{N}(\overline{\mathrm{KN}})$

K $\quad \mathrm{KNH}$

$\mathrm{N} \quad(\mathrm{K})$

$\mathrm{S} ? \quad \mathrm{~N}$

NW? $\overline{\mathrm{NH}}$

$\mathrm{W} ? \quad-\quad-$

SW

- $\quad 315$ Dichelachne

316. Deschampsia

317. Trisetum

318. Amphithromus

319. Danthonia

32c. (Eleusine)

32 I. Arundo

322. Triodia

323. Koeleria

324 Poa

325. Atropis

326. Festuca

327. Bromus

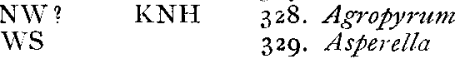

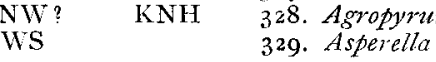

$\mathrm{KNH}$

$\mathrm{NH}$

NW

SW

NS?

$\mathrm{N}$

$\mathrm{S}$

W ? $\quad(\mathrm{K})$

$\mathrm{W} ? \mathrm{~S} \quad$ -

WS KII

W?

$\mathrm{W}$ ?

$\mathrm{N}$

NW? KNH

Looking at the map, it is evident that the western invasion most probably arrived by the ridge which reaches the western coast of $\mathrm{New}$ Zealand, and has two branches, one by Lord Howe Island, and one by Norfolk (the new map is a more recent one than the old, which shows the ridge not quite reaching Norfolk). The northern invasion is not so certain as to route. It may have passed by way of the Kermadecs, though we have already seen $(11$, p. 280) that this seems improbable; or it may have come by way of the belt of comparatively shallow water which unites the northern end of New Zealand with New Caledonia, and which passes through Norfolk Island. As this water is deeper than that over the western ridge, the northern may have been a more ancient invasion than the western, and there are other facts which point to the possibility of this, as we shall see in later papers. But in any case, northern, western, and Kermadec invasions all came from parts of 
Indo-Malaya, and there now exist on their probable tracks the islands of Lord Howe, Norfolk, and the Kermadecs.

One may go on to say at once, that while these islands show great traces of all these invasions, they do not by any means, even if all their floras be added together, contain the whole of the genera, or even of the wides, of those invasions. In actual fact, of the genera in the above list that

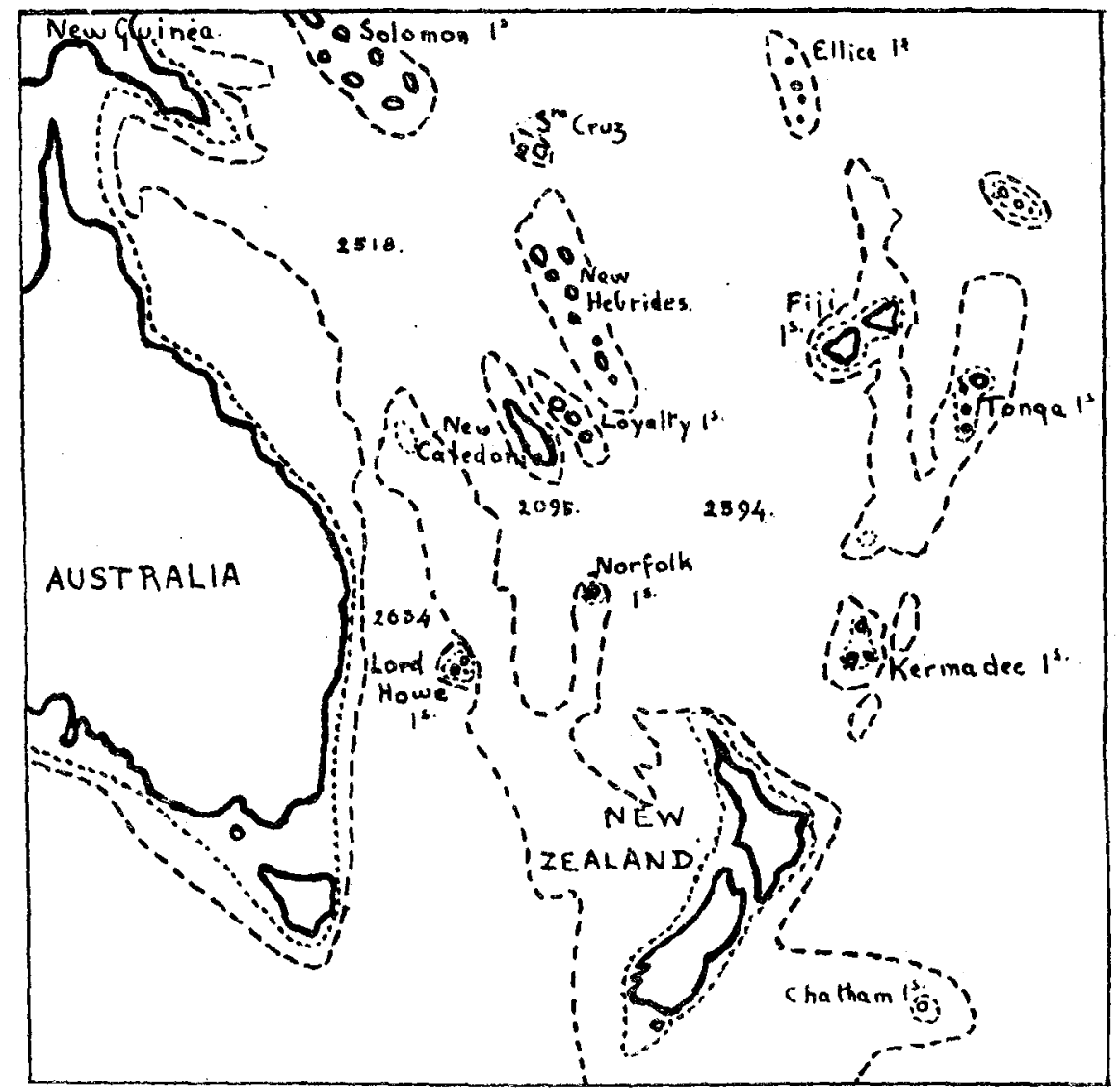

DIAGram 3. Soundings in the New Zealand area. ..... I00 fathoms. _... $-1,000$ fathoms. Numbers inserted here and there give the depth in fathoms at those points.

are marked $\mathrm{N}, \mathrm{W}, \mathrm{K}, \mathrm{NW}, \mathrm{NW}$ ?, NWS, NWS ?, N ?, NS?, WN, WN ?, WS, WS?, W? S?, KW, KN, or SW, they contain 82 out of $15^{2}$, or 54 per cent., and if the genera marked $W$ ? be added they contain 96 out of 219, or 44 per cent. Of the New Zealand wides of these genera $5^{\circ}\left(3^{8}\right.$ per cent.) occur in the islands (29 Dicotyledons and 2I Monocotyledons) and 8I do not $(33$ and 48$)$. One may feel inclined to say that this shows that transport must have been casual, but one must remember the very small area of these islands. Lord Howe is 7 miles long and has a maximum 
breadth of $\mathrm{I}$ mile, and Norfolk is only 5 miles long and a little broader, while the largest of the Kermadecs has an area of 12 sq. miles, and in all three cases the soil is purely volcanic. It would not, I think, be difficult to pick out of New Zealand, even in the centre, which is the richest area, a small area of uniform soil which would not contain more species than one of these islands. As a test, it is difficult to find an area of this size that has been sufficiently well botanized, but in the Flora of Cambridgeshire by Babington, I find that of 388 genera with $95^{\circ}$ species in the county, only 230 genera with $3^{88}$ species occur in the Wisbech district, which is about I5 miles by 10 with fairly uniform soil and conditions, though even there a number of coast (halophytic) species enter in one part. I do not, therefore, feel surprised that these little islands do not contain more of the northern and western invasions of New Zealand. It is worthy of a passing note that Lord Howe contains 160 species ( 52 locally endemic) and Norfolk 162 (43 locally endemic), and that these islands are of approximately the same area.

After these preliminary remarks, we may go on to the usual method of prediction and verification, and endeavour to see what can be made of the floras of these islands in this manner. For convenience in making predictions, we give below the complete list of the genera of these islands, with the islands $(\mathrm{K}, \mathrm{N}$, or $\mathrm{H})$ upon which they occur. The list is compiled from the Floras of Howe by Hemsley (3) and Oliver (5), Norfolk by Maiden (4), and the Kermadecs by Cheeseman (2).

TABLE III.

Flora of Lord Howe, Norfolk, and the Kermadec Islands.

Families and genera given in italics are found in New Zealand. Genera in capitals are endemic.

I. Ranunculaceae

No. of spp. Occur in

I. Clematis

2. Ranunculus 2. Magrzoliaceae

3. Drinyss 3. Menispermaceae

4. Stephania 4. Cruciferae

5. Nasturtium

6. Cardamine

7. Cakile

8. Lepidium 5. Capparidaceae

9. Capparis 6. Violaceae

10. Viola

11. Melicytus

12. Hymenanthera 13. Xylosma

$\begin{array}{ll}\text { I } & \text { NH } \\ \text { I } & \text { N } \\ \text { I } & \text { H } \\ \text { I } & \text { H } \\ \text { I } & \text { N } \\ \text { I } & \mathrm{K} \\ \text { I } & \text { N } \\ \text { I } & \text { H } \\ \text { I } & \text { N } \\ \text { I } & \text { N } \\ \text { I } & \mathrm{KN} \\ \text { 2 } & \mathrm{NH} \\ \text { I } & \text { H }\end{array}$

8. Pittospotaceat

14. Pittosporumti
9. Frankeniaceae

15. Frankenia Io. Maluaceae

I6. Malvastrum

17. Abutilon

I8. Hibiscus

I9. Lagunaria I1. Sterculiaceae

2o. Ungeria 12. Linaceae

21. Limum

13. Geraniaceae

22. Geranium

23. Pelargoniunt

24. Oxalis 14. Rutaceae

25. Melicope

26. Evodia

k k
No. of spp. Occur in

KNH

N

$\mathrm{N}$

N

NH

$\mathrm{N}$

N

$\mathrm{KN}$

NH

KH

KH

NH 


\begin{tabular}{|c|c|c|c|c|c|}
\hline & No. of $s p t^{\circ}$. & Occur in & & 10. of spp. & Cccur $21 \mathrm{t}$ \\
\hline $\begin{array}{l}\text { 27. Acronychia } \\
\text { 38. Xarthoxylum }\end{array}$ & 2 & NH & 75. Siegesbeckia & I & $\mathrm{K}_{\mathrm{H}}$ \\
\hline $\begin{array}{l}\text { 28. Xar.thoxylum } \\
\text { I5. Meliacieae }\end{array}$ & a & NH & $\begin{array}{l}\text { 76. Wedelia } \\
\text { 77. Cassinia }\end{array}$ & I & $\underset{\mathrm{H}}{\mathrm{NH}}$ \\
\hline 29. Dysoxylum & 2 & NH & 78. Bidens & I & KNH \\
\hline 16. Olacaceae & & & 79. Erechtites & 2 & $\mathrm{NH}$ \\
\hline 3о. Pennantia & I & $\mathrm{N}$ & 8o. Colula & I & $\mathrm{KNH}$ \\
\hline I 7 . Celastraceae & & & 81. Senecio & 2 & $\mathrm{KNH}$ \\
\hline 31. Elaeodendron & I & NHI & 82. Sonchus & $\mathrm{r}$ & $\mathrm{KNH}$ \\
\hline 32. Guioa Sapindaceac & 1 & & 83. Picris & 1 & $\mathrm{~N}$ \\
\hline $\begin{array}{l}\text { 32. Gitioa } \\
\text { 33. Dodonaea }\end{array}$ & I & $\mathrm{NH}$ & $\begin{array}{l}\text { 34. Goodenzaceae } \\
\text { 84. Scaevola }\end{array}$ & 2 & KH \\
\hline 19. Anacardiaceae & & & 35. Campanulaceae & & $\Lambda \Lambda$ \\
\hline 34. Corynocarpus & $\mathbf{r}$ & $\mathrm{k}$ & 85. Lobelia & I & $\mathrm{KNHI}$ \\
\hline 35. Coriaria $\begin{array}{l}\text { 20. Coriariacene } \\
\text { 35. }\end{array}$ & I & & 86. Wahlenbergia & I & $\mathrm{KNH}$ \\
\hline 2I. Legrminosae & 1 & $\mathbf{K}$ & 87. Leacopogon & I & $\mathbf{H}$ \\
\hline 36. Millettia & I & $\mathrm{N}$ & 88. Dracophyllhum & 1 & $\mathrm{H}$ \\
\hline 37. Glycine & I & $\mathrm{N}$ & 37. Plumbaginacene & & \\
\hline 38. Carmichaelia & 1 & $\mathrm{H}$ & 89. Plumbago & I & $\mathbf{N}$ \\
\hline 39. Mucuna & I & $\mathrm{H}$ & 38. Primulacede & & \\
\hline 40. Canavalia & I & KNII & 90. Sumolus & I & $\mathrm{kN}$ \\
\hline 4I. Vigna & I & $\mathrm{NH}$ & 39. Nyrsinaceae & & \\
\hline 42. Sophora & I & $H$ & 91. Rapanea & 4 & $\mathrm{KNH}$ \\
\hline 43. Caesalpinia & I & $\mathrm{NH}$ & 92. Aegiceras & 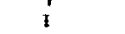 & \\
\hline 44. Clianthus & I & $\mathbf{N}$ & $\begin{array}{l}\text { 40. Sapolaceae } \\
\text { 93. Sideroxylon }\end{array}$ & 2 & \\
\hline 45. Acaena & I & $\mathrm{K}$ & $\begin{array}{l}\text { 93. Sideroxylon } \\
\text { 4I. Styracaceae }\end{array}$ & 2 & $\mathrm{NH}$ \\
\hline $\begin{array}{l}\text { 23. Saxifrasaceae } \\
\text { 46. COLMEIROA }\end{array}$ & 1 & H & $\begin{array}{l}94 \text { Symplocos } \\
\text { 42. Oleaceae }\end{array}$ & I & $\mathrm{H}$ \\
\hline 24. Haloragiaceae & $x^{*}$ & $\Pi$ & 95. Jasminum & $\mathbf{2}$ & NH \\
\hline 47. Haloragis & I & $\mathrm{K}$ & 96. Olea & 2 & $\mathrm{NH}$ \\
\hline $\begin{array}{l}\text { 49. Callitriche } \\
\text { 25. Myrtaceae }\end{array}$ & 1 & K & $\begin{array}{l}\text { 97. Notelea } \\
\text { 43. Apocynaceae }\end{array}$ & I & II \\
\hline 49. Metrosideros & 2 & $\mathrm{KH}$ & 98. Melodinus & I & $\mathrm{N}$ \\
\hline 50. Lepiospermutn & $\mathrm{I}$ & $\mathrm{H}$ & 99. Alyxia & 4 & NH \\
\hline 5. Melaleuca & I & II & 10o. Ochrosia & I & $\mathbf{H}$ \\
\hline 52. Acicalyptus & $\mathrm{I}$ & $\mathbf{H}$ & 10I. Lyonsia & I & $\vec{H}$ \\
\hline $\begin{array}{l}53 \text { Rhodomyrtus } \\
\text { 26. Lythraceae }\end{array}$ & I & $\mathbf{N}$ & $\begin{array}{l}\text { 44. Asclepiadacene } \\
\text { 102. Vincetoxicum }\end{array}$ & 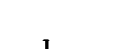 & \\
\hline 54. Lythrum & I & $\mathrm{N}$ & 103. Tylophora & 1 & $\begin{array}{l}\mathrm{H} \\
\mathrm{NH}\end{array}$ \\
\hline 27. Passifloracene & & & 104. Marsdenia & I & ${ }_{\mathrm{H}}^{\mathrm{NH}}$ \\
\hline $\begin{array}{l}\text { 55. Passiflora } \\
\text { 28. Cucurbitacease }\end{array}$ & 3 & NH & 45. Loganiaceae & & \\
\hline $\begin{array}{l}\text { 28. Cucurbitaceae } \\
\text { 56. Bryonopsis }\end{array}$ & $\mathbf{I}$ & $\mathbf{N}$ & $\begin{array}{l}\text { 105. Gemiostoma } \\
46 . \text { Gentianaceae }\end{array}$ & I & II \\
\hline 5. Sucyos & I & KNH & $\begin{array}{l}\text { 46. Gentianaceae } \\
\text { 106. Erythraea }\end{array}$ & 1 & $\mathrm{~N}$ \\
\hline 58. Melothria & I & & 47. Borasinaceae & 1 & $\mathbf{N}$ \\
\hline 29. Ficoideae & & & 107. Cynoglossum & I & $\mathrm{N}$ \\
\hline $\begin{array}{l}\text { 59. Mesembryanthemum } \\
\text { 6o. Tetragonia }\end{array}$ & $\begin{array}{l}2 \\
2\end{array}$ & KNH & 48. Convolvulaceae & & \\
\hline 61. Sesuvium & I & $\begin{array}{l}\mathrm{KNH} \\
\mathrm{H}\end{array}$ & I08. Ipomoea & 5 & KNH \\
\hline 30. Umbelliferae & & & $\begin{array}{l}109 \text { Calystegia } \\
40 . \text { Solanaceae }\end{array}$ & 4 & KNH \\
\hline $\begin{array}{l}\text { 62. Hydrocotyle } \\
\text { 63. Apium }\end{array}$ & 2 & KH & ro. Solanum & 3 & KNII \\
\hline $\begin{array}{l}\text { 3I. Araliaceae } \\
\text { 64. Nothopanax }\end{array}$ & 2 & $\mathrm{KNH}$ & $\begin{array}{l}\text { 50. Scrophulariaceae } \\
\text { I11. Veronica }\end{array}$ & 2 & $\mathrm{KN}$ \\
\hline 65. Meryta & $\begin{array}{l}2 \\
2\end{array}$ & $\underset{N}{\mathrm{KH}}$ & 5I. Gesneriaceae & & \\
\hline 66. Randia & & & 112. $N E G R I A$ & I & $\mathrm{H}$ \\
\hline $\begin{array}{l}\text { 66. Randia } \\
\text { 67. Psychotria }\end{array}$ & 1 & $\mathrm{H}$ & 52. Bignoniaceae & & \\
\hline $\begin{array}{l}\text { 67. Psychotria } \\
\text { 68. Coprosma }\end{array}$ & I & $\mathrm{H}$ & 113. Tecoma & 2 & NII \\
\hline 68. Coprosma & 8 & $\mathrm{KNH}$ & 53. Acanthaceae & & \\
\hline $\begin{array}{l}\text { 33. Compositae } \\
\text { 69. Ageratum }\end{array}$ & & & II4. Eranthemum & 1 & $\mathbf{H}$ \\
\hline $\begin{array}{l}\text { 69. Ageratum } \\
\text { 7o. Lagenophora }\end{array}$ & $\cdot \vec{I}$ & $\begin{array}{l}\mathbf{K} \\
\mathbf{K}\end{array}$ & 54. Myoporaceae & & \\
\hline $\begin{array}{l}\text { 70. Lagenophora } \\
\text { 71. Vernonia }\end{array}$ & I & $\stackrel{\mathbb{N}}{\mathrm{N}}$ & I15. Myoporum & 3 & KNH \\
\hline 72. Brachycome & I & $\mathrm{H}$ & $\begin{array}{l}\text { I6. Verbenaceae } \\
\text { I } 5 \text { erbena }\end{array}$ & $\mathbf{I}$ & $N$ \\
\hline 73. Olearia. & 2 & $\mathrm{H}$ & I17. Vitex & I & $\mathrm{N}$ \\
\hline 74. Gnaphalium & 3 & KNH & 118. Avicennia & 1 & $\mathbf{H}$ \\
\hline
\end{tabular}


No. of spp. Occur ine

56. Labiatae

119. Westringia

1 20. Plectranthus 57. Plantaginacae

12 I. Plantago 58. Nyclaginaccae

1 22. Boerhaavia

123. Pisonia 50. Amarantaceae

124. Achyranthes 6o. Chenopodiaceae

I 25. Rhagoulia

126. Atriplex

127. Salicornia 61. Polygonaceae

128. Rumex

129. Muehlonbeckia 62. Piperaceae

I30. Macropiper

I31. Peperomia 63. Chloranthaceat

132. Ascarina 64. Lanraceae

133. Cryptocarya 65. Thymelaeaceae

134. Pinuelea

135. Wikstroemia 66. Loran'haceac

136. Koithalsella 67. Santalaceae

137. Exocarpus 68. Euphorbiacae

138. Alenrites

139. Homalanthus

140. Euphorbia

I 4 '. Hemicyclia

I 42. Baloghia

143. Excoecaria 69. Urticaceae

144. Celtis

I.45. Malaisia

146. Ficus

147. Psendomorus

I48. Procris

I 49. Elatostema

I50. Boehmeria

151. Parietaria 7o. Hydrocharidaceae

I52. Halophila 71. Orchidaceae

153. Oberonia

154. Dendrobium

I55. Buibophyllum

156. Phreatia

157. Microtis

158. Acianthus

159. Cleisostoma 160. Moraea

$$
\text { 72. Iridaceae }
$$

(I) If, as I maintain, these islands lie upon the tracks of previovis plantinvasions of New Zealand by land, then one will expect to find that a large part of their flora consists of families, genera, and species which also occur in New Zealand.

206. Triticum
73. Amaryllidaceae

No. of spp. Occur in

$\mathrm{H}$
$\mathrm{H}$
$\mathrm{H}$
$\mathrm{NH}$
$\mathrm{NH}$
$\mathrm{KH}$
$\mathrm{H}$
$\mathrm{H}$

161. Crinum

74. Liliaceae

162. Smilax

163. Rhipogonum

164. Geitonoplesium

165. Cordyline

166. Phormium

167. Dianella

75. Commelinaceae

168. Commelina

76. Flagellariaceae

169. Flagellaria

77. Juncaceae

$\mathrm{KNH}$

I 7o. Juncus

I71. Luzula

78. Palmae

KNII 172. Rhopalostylis

KNH

173. HED YSCEPE

174. Clinostigma

K

$\mathrm{H}$

II

$N$

$\mathrm{NH}$

NH

I5. HOWEA

79. Pandanaceae

176. Pandanus

17\%. Freycinetia

8o. Typhaceae

Ij8. Typha

81. Araceae

I 79. Colocasia

82. Cyperaceae

180. Cyperus

181. Mariscus

182. Kyllinga

$\begin{array}{ll}\text { KH } & \text { 183. Cladium } \\ \text { NH } & \text { I8. Eleocharis }\end{array}$

$\mathrm{H}$ 185. Gahnia

NH 186. Uncinia

N 187. Scirpus

188. Carex

NH 83. Gramineae

NH 189 . Oplismenus

190. Spinifex

191. Paspalum

192. Panicum

I93. Imperata

194. Cenchrus

195. Andropogon

I96. Microlaena

197. Echinopogon

198. Sporobolus

199. Calamagrastis

200. Dichelaihne

20r. Phragmites

202. Cynodon

203. Eleusine

204. Foa

205. Agropyrum
N

INH

$\mathrm{N}$.

$\mathrm{NH}$

$\mathrm{N}$

NH

NH

H

H

KN

H

H

H

$\mathbf{N}$

KN

N

N

NH

H

N

$\mathbf{H}$

KNH

KNH

KNH

$\mathrm{H}$

NH

$\mathrm{KN}$

K

$\mathbf{K}$

$\mathbf{N}$

$\mathbf{N}$

NH

KNH

NH

$\mathrm{H}$

N

K

KNH

N 
TABLE IV.

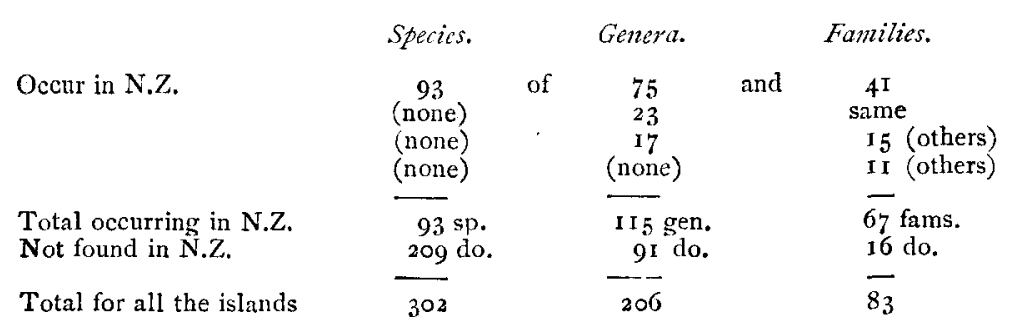

Thus nearly one-third of the species, more than half of the genera, and fourfifths of the families occur in New Zealand.

(2) One will expect the 23 genera of the same families to be chiefly represented by endemic forms, or by Australian or Polynesian wides which have been too late to reach New Zealand. In actual fact the 23 genera are represented in the islands by 3 I species, of which $2 \mathrm{I}$ are endemic to the islands, I (Imperata Cheesemani) to the Kermadecs, 8 to Norfolk, I I to Howe, and I (Mariscus haematodes) to both Norfolk and Howe. A further 7 species are Australian wides, 2 are Polynesian wides, and I (Nasturtium sylvestre) is probably introduced. The prophecy is thus fully borne out.

(3) One will also expect the I 7 New Zealand genera, belonging to the I 5 families which are not represented in New Zealand by any species that occur on these islands, to be similarly made up of endemic species and Australian and Polynesian wides. In actual fact these $I 7$ genera show on the islands 26 species, which are composed of 18 endemics, 7 Australian wides, and I Polynesian wide.

(4) The I I New Zealand families, which are not represented on the islands either by genera or by species that occur in New Zealand, are Saxifragaceae, Goodeniaceae, Apocynaceae, Gentianaceae, Boraginaceae, Gesneriaceae, Labiatae, Amarantaceae, Lauraceae, Iridaceae, and Amaryllidaceae, and are represented on the islands, as one would rather expect, by II endemic species, including two endemic genera (Colmeiroa and Negria) and ro Australian wides. One of the endemic species, Moraea Robinsoniana, belongs to an African genus, which probably arrived by sea carriage.

(5) Passing on now to the species, genera, and families given in Table IV as not found in New Zealand, one will expect that a large proportion of the species will be endemic in the islands. In fact, of the 209 species that occur on the islands and have not been found in New Zealand, no less than ${ }_{0}{ }_{5}$ appear to be endemic to the islands.

(6) One will expect that the remaining 104 species will be Australian or Polynesian wides, especially the former. In fact 93 of them are Australian and 9 Polynesian, while 2 (Nasturtium syluestre and Cakile maritima) are perhaps introduced, being European types. 
(7) One will also expect to find that the 209 species that do not reach New Zealand will be largely members of genera and still more of families that do reach it. Those families and genera that reach New Zealand will on the whole be the earliest arrivals of their respective affinity groups. These groups will thus, so to speak, have the start of the others, and there will be more likely to be more of them in the islands than of other groups, of which none have reached New Zealand. Testing this we find in the island flora :

\section{TABLE V.}

Not reaching N.Z.

Found in N.Z. of these

Leaving

$$
\begin{array}{ccccc}
209 \text { species of } 155 & \text { genera and } 74 \text { families } \\
\text { (none) } & 64 & , & \text { (with I } 30 \text { spp.) and } 58 \text { families } \\
& 9 \text { I } & \text { " } & \text { I I } 2, ", \quad \text { I } 6
\end{array}
$$

(8) One will expect to find the families and genera that have reached New Zealand better represented in the islands, on the whole, as being older, than those that have not.

\section{TABLE VI,}

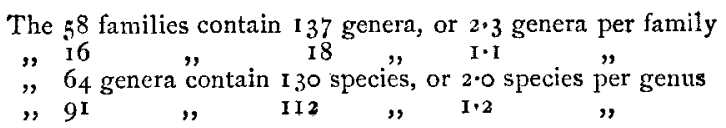

(9) The' 6 families that do not reach New Zealand at all, are all, as one would expect (they being probably young, and therefore on the average small, in the islands), small and not widely distributed in the islands. They are Menispermaceae (1 sp. Howe), Capparidaceae (I Norf.), Bixaceae (I Howe), Frankeniaceae (I Norf.), Sterculiaceae (I Norf.), Celastraceae (I Norf., I Howe), Lythraceae (I Norf.), Plumbaginaceae (I Norf.), Styracaceae (1 Howe), Asclepiadaceae ( 2 on Howe only I Norf., I Howe), Bignoniaceae (I Norf., I Howe), Acanthaceae (I Howe), Hydrocharidaceae (I Howe), Commelinaceae (I Norf,, I Howe), Flagellariaceae (I Howe), Araceae (I Norf.). All are small, and little distributed among the islands. Similarly, of the 91 genera, 65 have only one species reaching one island, Io one species reaching two.

(Io) Of the 9I genera that do not occur in New Zealand, one will expect the greater proportion (both absolute and per family) to belong to families that have reached New Zealand, for these will be the older families in their affinity circles. In actual fact 73 belong to 42 families that have reached New Zealand, or four-fifths of the total, and $\mathrm{I} \cdot 7$ genera per family, while 18 belong to 16 families that have not reached New Zealand, or one-fifth of the total, and I.I genera per family. The prophecy is thus borne out.

(11) To turn now to another type of consideration, one will expect the bulk of the floras of these islands to belong to genera and families that in Table II are marked as probably belonging to northern, Kermadec, or 
western invasions of New Zealand. In actual fact, of the I I 5 genera that reach New Zealand, we find that they are marked as follows :

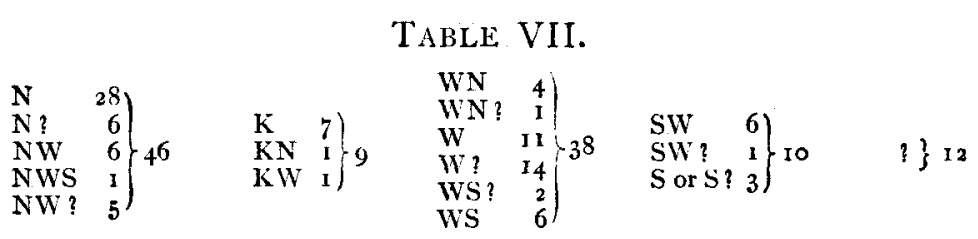

Thus 55, or just less than half, are of unquestionably northern origin ( $\mathrm{N}$ or $\mathrm{K}$ ) as judged simply by their distribution in New Zealand, and $3^{8}$ more are probably western, while I 2 have so few species, and so widely distributed in New Zealand, that one cannot tell from their local distribution by which route they may have entered.

There remain 10 genera, marked in Table II as SW or S. The six SW genera are represented in the islands by (i) Lepidium Howeiinsulae, endemic and allied to L. oleraceum of New Zealand, therefore probably derived from New Zealand; (ii) Brachycome segmentosa, endemic and allied to an Australian species; (iii) Cotula australis, found in all three islands, and in New Zealand down to Foveaux Strait, as well as in Australia, and therefore probably an Australian species which entered by the west; (1v) Plantago Headleyi (Howe), endemic and near $P$. aucklandica of New Zealand, and therefore probably derived from New Zealand; (v) Uncinia filiformis (Howe), found in New Zealand from 280 miles south of N. Cape down to Stewart Island, and therefore possibly a western type; also found in Australia; and (vi) Carex semiforsteri (Kermadecs), found down to Foveaux Strait in New Zealand and thus probably not southern, $C$. Neesiana, endemic in Norfolk, C. inversa (Norfolk), down to Foveaux Strait and in Australia, and therefore (on both counts) probably western, $C$. breviculmis, the same, and $C$. gracilis, otherwise found in Australia only.

The one genus marked SW? is Ramunculus, which is represented in Norfolk by $R$. parviflorus, an Australian species that does not reach New Zealand.

The three southern genera are (i) Gardamine, represented in the Kermadecs by $C$. stylosa, found from 60 to 820 miles in New Zealand, and therefore probably western; also found in Australia; (ii) Veronica, represented by $V$. calycina, an Australian species, in Norfolk, and by $V$. salicifolia in the Kermadecs, a very widely spread New Zealand type which probably reached the Kermadecs early; and (iii) Luzula, represented in Howe by the endemic L. longiflora, allied (?) to the New Zealand antarctic island L. crinita.

(12) The Kermadecs, Norfolk, and Howe contain a large number of endemic forms. It is clear that on the hypothesis of age and area, these should occur principally in the families and genera which have been in the 
islands for the longest time, i. e. in those which are the most likely to have reached New Zealand. The endemics are:

TABLE VIII.

The letters $\mathrm{K}, \mathrm{N}, \mathrm{H}$ refer to the islands in which the families or species are found.

I. Magnoliaceae (NH)

I. Drimys Hozeana

2. Cruciferae (KNH)

2. Lepidium Howeitusulae

3. Violaceae (KNH)

3. Hymenanthera latifolia

4. Pittosporaceae ( $\mathrm{KNH}$ )

4. Pittosporum bracteolatum

5. erioloma

5. Malvaceae ( $\mathrm{NH}$ )

6. Abutilon Julianae

7. Hibiscus insularis

6. Sterculiaceac $(\mathrm{N})$

8. Ungeria floribunda

7. Rutaceae (KNH)

9. Melicope contermina

1o. Evodia polybotrya

II. littoralis

12. Aeronychia Endicheri

8. Meliaceae (NH)

13. Dysoxylum Patersonianum

14. pachyphyllum

9. Celastraceae (NH)

5. Elacodendron curtipendulum

Io. Sapindaceae (H)

16. Guioa coriaiea

1 I. Leguminosae (KNH)

17. Carmichaelia exsul

18. Milletia australis

19. Clianthus Baueri

12. Saxifragaceae $(\mathbf{H})$

20. COLAEIROA CARPODETOIDES $\mathrm{H}$

I3. Myrtaceae (KNH)

2 [. Acicalyptus Fullagari

22. Metrosideros neroulosa

14. Passifloraceae (NH)

23. Passiflora Baueriana

24.

glabra

I. . Cucurbitaceae (KNIH)

25. Bryonopsis afinis

26. Melothria Baueriana

16. Araliaceae (KNH)

27. Nothopanax cissodendron

28. Meryta latifolia

29. anyustifolia

77. Rubiaceae (KNH)

3o. Randia stipulosa

31. Psychotria Carronis

32. Coprosma prisca

33 .

34.

35 .

36.

37.

lanceolaris

putida

pilosa

petiolata acutifolia

I8. Compositae (KNH)

$3^{8}$. Brachycome segmentosa

39. Olearia Balli

40. Mooneyi

41. Cassinia tenuifolia

42. Senticio insularis
H

H

$\mathrm{N}$

$\mathrm{N}$

II

$\mathrm{N}$

$\mathrm{N}$

$\mathrm{N}$

II

H

$\mathrm{N}$

$\mathrm{N}$

$\mathrm{N}$

$\mathrm{H}$

$\mathrm{N}$

H

H

$\mathrm{N}$

$\mathrm{N}$

$\mathrm{H}$

$\mathrm{H}$

H

$\mathrm{N}$

$\mathrm{N}$

$\mathrm{N}$

N

$\mathrm{H}$

$\mathrm{N}$

$\mathrm{N}$

$\mathrm{H}$

$\mathrm{H}$

H

$\mathrm{H}$

H

$\mathrm{N}$

$\mathrm{K}$

$\mathrm{H}$

$\mathrm{H}$

$\mathrm{H}$

H

II
I 9. Goodeniaceae (KH)

43. Scaevola gracilis

20. Epacridaceae $(\mathbf{H})$

44. Dracophyllum Fitzgeraldi

21. Myrsinaceae (KNH)

45. Rapanea kermadecensis

46. platystigma

47. mytilina

22. Sapotaceae (NH)

48. Sideroxylon Howeanum

23. Styracaceae (H)

49. Symplocos candelabrum

24. Oleacene ( $\mathrm{NH}$ )

5o. Nolelaea quadristaminea

25. A pocynaceae ( $\mathrm{NH}$ )

51. Alyxia Lindit

52. squamulosa

53. gynopogon

54. Melodinus Baueri

26. Asclepiadaceae (NH)

55. Tylophora biglandulosa

27: Loganiaceae (H)

56. Geniostoma petiolosum

28. Convolvulaceae (KNH)

57. Ipomoea cataractae

58. Calystegia affinis 29. Solanaccae (KNH)

59. Solanum Bauerianum

3o. Gesneriaceae (H)

6o. NEGRIA RHABDOTHAMNOIDES

3I. Myoporaceae (KNH)

61. Myopomem obscurum

32. Plantaginaceae $(\mathrm{H})$

62. Plantugo Headleyi

33. Amarantaceae (NH)

63. Achyranthes arborescens

34. Piperaceae (KNH)

64. Peperomia Baueriana

35. Chloranthaceae (K)

65. Asiarina lanceolata

36. Lauraceae $(\mathrm{H})$

66. Cryptocarya Gregsoni

37. Thymelaeaceae (NH)

67. Pimelea congesta

68. Wikstroemia australis

38. Santalaceae (NH)

69. Exocarpus homalo lada

70.

phyllanthoides

$\mathrm{NHI}$

39. Euphorbiacene (KNH)

71. Euphorbia obliqua

72. Norfolkiana

73. Homalanthus polyandrus

40. Urticaceae (KNH)

74. Celtis ambiyphylla

75. Ficus columnaris

76. Procris montana

77. Bochmeria dealbata

78.

79 .

australis

calophletia
K

H

K

$\mathbf{H}$

H

H

HI

II

H

$\mathrm{H}$

$\mathrm{N}$

$\mathrm{N}$

II

$\mathrm{N}$

$\mathrm{N}$

N

H

$\mathrm{N}$

H

$\mathrm{N}$

N

K

II

$\mathrm{H}$

$\mathbf{N}$

$\mathrm{H}$

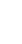

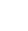

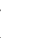

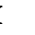

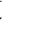

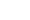

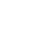

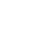

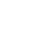

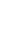

N

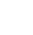

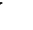

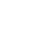

.

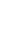

I

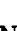

N

$\mathbf{K}$

H

H

N

K

$\mathrm{N}$

H 
Willis.-Plant Invasions of Nerv Zealand.

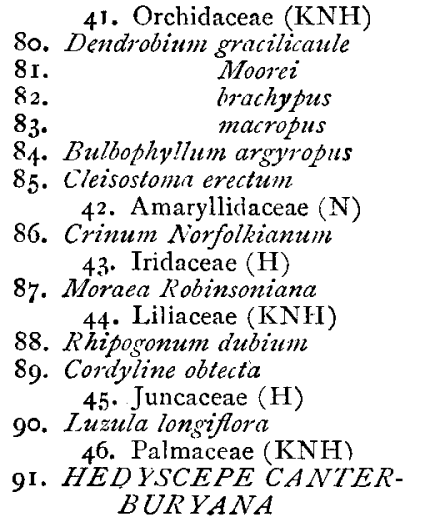

\begin{tabular}{|c|c|c|}
\hline & 92. Clinostigma Mooreanum & 1 \\
\hline $\mathrm{H}$ & 93. HOWEA BELMOREANA & $\mathrm{H}$ \\
\hline $\mathrm{H}$ & 94. FORSTERIANA & $\mathbf{H}$ \\
\hline $\mathrm{N}$ & 95. Rhopalostylis Baueri & N \\
\hline $\mathrm{N}$ & 96. Cheesemanii & \\
\hline $\mathrm{N}$ & 47. Pandanaceae $(\mathrm{NH})$ & \\
\hline $\mathrm{H}$ & 97. Pandanus Forsteri & \\
\hline $\mathrm{N}$ & $\begin{array}{l}\text { 98. Freycinetia Baueriana } \\
\text { 48. Cyperaceae (KNH) }\end{array}$ & \\
\hline & 99. Mariscus haematodes & $\mathrm{NH}$ \\
\hline H & roo. Cladium insulare & \\
\hline & 101. Carex Neesiana & \\
\hline $\mathrm{N}$ & 49. Gramineae (KNH) & \\
\hline $\mathrm{N}$ & I02. Imperata Cheesemanii & \\
\hline & 103. Panicum Norfolkiamum & \\
\hline $\mathrm{H}$ & 104. Poa polyphylla & \\
\hline & 105. Triticum Kingianum & \\
\hline
\end{tabular}

These sum up to 105 species, belonging to $8 \mathrm{I}$ genera and 49 families. Of these 63 belong to 44 genera and 33 families which occur in New Zealand, while 38 species of 33 genera more belong to families that occur (though not the genera) in New Zealand. Thus, as predicted, the greater proportion occur in genera that have reached New Zealand, whilst nearly all the families that contain endemics have also reached that country, including the families (Saxifragaceae, Gesneriaceae, Palmaceae) that contain the endemic genera (Colmeiroa, Negria, Hedyscepe, Howea).

The families that contain endemics and do not reach New Zealand are Sterculiaceae, Celastraceae, Styracaceae(Symplocaceae), and Asclepiadaceae, each with one endemic species only, and represented, the first two each by the endemic species only in Norfolk Island, the third by the endemic only in Howe, and Asclepiadaceae by the endemic Tylophora in both Norfolk and Howe, and by Vincetoxicum carnosum and Marsdenia rostrata, both Australian species, in Howe.

The genera that contain endemics and do not reach New Zealand belong, all but these four, to families that reach New Zealand. They are Abutilon, Ungeria, Evodia," Acronychia, Elaeodendron, Millettia, Guioa, COLMEIROA, Acicalyptus, Bryonopsis, Melothria, Randia, Psychotria, Scaevola, Symplocos, Notelaea, Alyxia, Melodinus, Tylophora, NEGRIA, Achyranthes, Cryptocarya, Wikstroemia, Baloghia, Homalanthus, Celtis, Ficus, Procris, Cleisostoma, Crinum, Moraea, HED YSCEPE, Clinostigma, HOWEA, Pandanus, Panicum, Triticum. Most are represented by one species only, but Evodia has two, Alyxia three, and Boehmeria three.

(I3) It is evident, on my hypothesis, that the endemics should belong to the families that have been longest in the islands, i.e. on the whole to the largest families. In actual fact they belong to 49 families with I 57 genera, or $3^{.2}$ genera per family, while the families that contain no endemics are 34 with 49 genera, or $x \cdot 4$ genera per family. Twenty-three families with 3 or more genera contain 64 of them, 16 with 2 genera contain 16 , and 44 with one genus contain 25 only. 
(14) In the same way they should belong to the larger genera of the island floras. In actual fact, they belong to $8 \mathrm{I}$ genera which contain altogether 150 species, or $1 \cdot 8$ species per genus. The genera that contain no endemics are 125 with $I 52$ species, or $I \cdot 2$ species per genus.

(I5) One will expect the island genera which contain endemics, nasmuch as they will on the whole be old, to be fairly large in New Zealand (when they reach there). There are $8 \mathrm{r}$ in all of them, and 37 do not reach New Zealand. The remaining 44 contain in New Zealand 362 species, or an average of 8.2 species per genus, while the average size of a genus in New Zealand is only 4.2 species. Even if the $3^{62}$ be divided by the whole $8 \mathrm{r}$, the result is $4 \cdot 4$.

It is worthy of note, with reference to Professor Sinnott's hypothesis of 'swamping' (6, p. 214), that of the 362 species in New Zealand only 38 are wides. But on the other hand, the genera which are represented by wides in the islands are usually represented by wides in New Zealand.

(I6) One will expect the families with endemics in the islands to be on the whole the same as the families with endemics in New Zealand, being the older families in each case. In New Zealand 76 families out of $9 \mathrm{r}$ contain endemics, but of these $18(10$, p. 359$)$ are chiefly southern. In the islands 49 families contain endemics, and of these 38 are the same as in New Zealand. The other II include Sterculiaceae, Celastraceae, Styracaceae, and Asclepiadaceae, which do not occur in New Zealand, and also Cucurbitaceae, Goodeniaceae, Sapotaceae, Convolvulaceae, Amarantaceae, Piperaceae, and Amaryllidaceae, 7 families with only 17 species in all, and ${ }_{5} 5$ of them wides, while the average range in New Zealand is only $5^{87}$ miles (against a possible range of $\mathrm{I}, 080$ ), all of them facts which point to the probably (comparatively) recent arrival of these families in New Zealand.

(I7) In the same way, the genera with endemics in the islands should in general be genera that have endemics in New Zealand, or genera probably only comparatively recently arrived there. Of the $8_{I}$ island genera with endemics, 37 do not occur in New Zealand, leaving 44 that do, of which no fewer than 38 possess endemics in New Zealand. It may be said, by those who have not worked at the matter in detail, that this is probably only the normal percentage of New Zealand genera that possess endemics, but in actual fact 95 genera out of 321 have no endemics in New $Z$ ealand, or a much higher percentage. The 6 genera that have no endemics in New Zealand are Hibiscus, Ipomaea, Calystegia, Solanum, Peperomia, and Euphorbia, which have in all 12 wides with an average range of 647 miles, and are thus small and slightly ranging genera, therefore probably on the whole young in New Zealand (in their affinity groups).

. (I8) One will expect the endemics of the islands to belong chiefly to the families that reach most islands. 
TABLE IX.

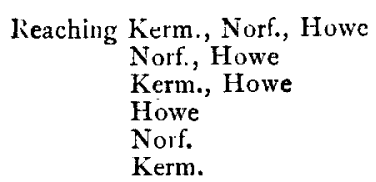

\begin{tabular}{|c|c|c|}
\hline 22 fams. & 50 gen. & $69 \mathrm{spp}$ \\
\hline $\left.\begin{array}{r}13 \\
I\end{array}\right\} 14$ & $17\} 18$ & $\left.\begin{array}{r}22 \\
1\}\end{array}\right\}$ \\
\hline 10$)$ & 10 j & 10) \\
\hline $\left.\begin{array}{l}2 \\
1\end{array}\right\}^{I} 3$ & $\left.\begin{array}{l}2 \\
I\end{array}\right\}^{13}$ & $\left.\begin{array}{l|l}2 & 13 \\
1\end{array}\right\}^{3}$ \\
\hline
\end{tabular}

Thus much the best representation is among the families that reach all three groups of islands, the second best among those reaching two.

(19) Turning now to more general predictions, one will expect that the largest families in the islands will be those reaching three groups, then those reaching two, and lastly those reaching one only.

TABLE X.

\begin{tabular}{|c|c|c|c|c|c|c|}
\hline Reaching & No. of fants. & & 2 & & & Containing \\
\hline Three groups & 27 & 3 & 8 & 5 & 2 & $5,6,6,7,8,9,9,15,18$ \\
\hline Two gioups & 24 & 13 & 5 & 4 & 2 & \\
\hline One group & $3^{2}$ & 23 & 4 & - & -. & \\
\hline
\end{tabular}

Thus the families reaching three groups are 27 with 125 genera, or an average of 4.7 , those reaching two are 24 with 43 (average $I \cdot 8$ ), those reaching one are 32 with 36 (average $\mathrm{I} \cdot \mathrm{I}$ ).

(20) One will expect the same of the genera. In fact the 29 genera that reach three groups contain 73 species, or 2.5 per genus, while the 54 that reach only two groups contain 97 species ( 1.8 per genus) and the 123 that only reach one contain I 32 species, thus completely fulfilling the prediction.

(2I) Again, one will expect the families that reach three groups of islands, as oldest in their various affinities, to be best represented in New Zealand. The 27 families that reach all three groups of islands are represented in New Zealand by 79 out of II 5 genera that reach New Zealand, the remaining $5^{6}$ families only containing $3^{6}$ genera.

(22) In the same way, the genera reaching all three groups of islands should be best represented in New Zealand. There are 30 of these, and 28 of them are represented in New Zealand, while of the remaining 176 only 87 are represented. The two genera reaching all three groups of islands and not reaching New Zealand are Cancvalia, represented in all by C. obtusifolia, one of the stock plants of the drift of sea currents, and which one would expect in New Zealand, and Boehmeria, represented by a separate endemic in each of the three groups of islands.

(23) The species in common between three groups of islands, as oldest, should show the largest proportion of wides, and then those occurring in two or one groups. 
TABLE XI.

$\begin{array}{lcc}\text { Occutring in } & \text { Wides. } & \text { Endemi:s. } \\ \text { Three groups } & 23(100 \%) & - \\ \text { Two groups } & 35(90 \%) & 4 \\ \text { One group } & 139(58 \%) & \text { 101 }\end{array}$

Looking at the results that have been set forth in this paper, one is rather inclined to come to at least one important general conclusion. From the fact that by using age and area as a basis, 23 successful predictions have been made, many of which involve the mutual relationships of these islands and New Zealand in the matter of floras and their distribution, one may reasonably, I think, infer that age and area is as valid for these islands as for the mainland of New Zealand, not only as regards their local flora (considered entirely by itself) but as regards their floral relationships with New Zealand. But if the latter be the case, then it is clear that in all probability these islands must once have been a part of, or close to, the land communications over which the Indo-Malayan invasions of New Zealand travelled (cf. 5, p. I I 2). It is almost impossible to imagine them receiving by casual transport across the water a flora which would show such striking numerical relationships, and relationships in such detail, to the flora of New Zealand. One cannot imagine that age and area should hold for the mutual relationships of two areas that were peopled with plants by independent invasion of casual waifs across the ocean. Taking these facts together with those set forth for New Zealand on p. 473, above, one may, I think, come to the conclusion that the peopling of New Zealand from Indo-Malaya was by land (not, of course, necessarily absolutely continuous, but with at most comparatively narrow straits), and that the islands which have been dealt with in this paper formed part of, or were very near to, that land.

I am much indebted to the Director of Kew Gardens, Sir David Prain, C.M.G., F.R.S., for references to literature, and to my daughter Margaret for Diagram 3 .

\section{SUMMARY.}

In this paper age and area is applied to the floras of the islands outlying between New Zealand and the nearest larger areas of land to the north or north-west. It proves to be equally applicable to them, both for themselves and for their relationships to New Zealand, showing that they must in all probability have formed part of a land mass or masses running down to New Zealand from Indo-Malaya. Lists are given of the New Zealand genera (Table II), showing the invasions of which each may be supposed to have formed a part, deduced from their local distribution in New Zealand; of the genera of the islands (Table III), showing those that reach New Zealand; and of the endemics of the islands (Table VIII). It is shown that the 
invasions of New Zealand were probably four-northern, Kermadec, western, and southern-the western probably arriving by the ridge on which stand Norfolk and Lord Howe.

Applying the usual method of prediction and verification, it is then shown that (I) a large part of the floras of these islands also occurs in New Zealand; (2), (3), and (4), genera and families not represented in New Zealand by actual species, are represented by endemics or by Australian and Polynesian wides in the islands; (5) more than half the species not found in New Zealand are endemic to the islands, and (6) the rest are Australian and Polynesian wides; (7) these species belong very largely to genera and families that do reach New Zealand; (8) families and genera that have reached New Zealand are better represented on the islands than those that have not; (9) the families that do not reach New Zealand at all are all small and little distributed in the islands ; (Io) the genera that do not reach New Zealand belong mainly to families that do; (II) the bulk of the island floras belongs to genera and families of the northern, Kermadec, and western invasions of New Zealand; (12) the island endemics occur chiefly in genera and families that have reached New Zealand; they occur chiefly in (I3) the larger families of the islands, and (14) the larger genera; (15) the island genera with endemics are usually large in New Zealand; ( 16 and 17 ) the island families and genera with endemics are in general the same as in New Zealand; (I 8) the island endemics belong chiefly to the families that reach three islands; (I9) the largest families in the islands are those reaching all three, and (20) similarly the largest genera; $(2 \mathrm{I})$ the families that reach three are best represented in New Zealand, and (22) similarly the genera; $(23)$ the species in common between three islands show the largest proportion of wides, then those from two and from one.

\section{LITERATURE.}

1. Arber: On the Law of Age and Area, in relation to the Extinction of Species. Ann. of Bot., xxxiii, 19I9, p. 21 I.

2. Cheeseman: Flora of New Zealand. Wellington, igo6.

3. Hemstery: Flora of Lord Howe Island. Ann. of Bot., x, i 896, p. $22 \mathrm{x}$.

4. Matden: Flora of Norfolk Island. Journ. Linn. Soc., N.S.W., xxviii, 1903, p. 692.

5. Or.Iver: Vegetation and Flora of Lord Howe Island. Trans. and Proc. N.Z. Inst., xlix, I91 7, p. 94 .

6. Sinno't : The Age and Area Hypothesis, \&c. Ann. of Bot., xxxi, 1917, p. 209.

7. Willts : On Ceylon. Phil. Trans., B., ccvi, s9i5, p. 307 ; Proc. Roy. Soc., B., lxxxix, I916; and Ann. of Bot. xxx, 1916, p. 1.

8. - : On New Zealand, eight papers in Ann. of Bot., 1916-19, especially-

9. - : Distribution of Species in N.Z, xxx, 1916, p. 437.

10. - Scurces and Distribution of the N.Z. Fiora, xxxii, 1918, p. 339.

11. - F : Foras of the Outlying Islands ..., xxxiii, 1919, p. 267. 